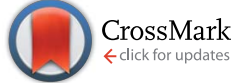

Cite this: RSC Adv., 2016, 6, 24667

Received 22nd January 2016 Accepted 25th February 2016

DOI: $10.1039 / \mathrm{c} 6 \mathrm{ra01989g}$

www.rsc.org/advances

\title{
Full graphitization of amorphous carbon by microwave heating $\dagger$
}

\author{
Teawon Kim, ${ }^{a}$ Jaegeun Lee ${ }^{b}$ and Kun-Hong Lee*a
}

Natural graphite is labelled as a supply risk material due to rapidly increasing demand and limited reserves. The conventional method for the production of synthetic graphite has relied on the thermal heating at an extremely high temperature, $3000{ }^{\circ} \mathrm{C}$, and long processing time, typically 2 weeks. Here, we report a novel and efficient method of graphitization using microwave heating with metal catalysts. The amorphous carbon powders turned into crystalline graphite in 5 minutes. Ideas for the scale-up of this work were proposed. In addition, numerical analysis revealed that the Maxwell-Wagner-Sillars polarization is inadequate for the mechanism underlying the microwave heating of solid carbon materials.

\section{Introduction}

Although academic interests have been moved to novel nano-carbons, graphite still dominates the commercial market by an incomparable scale.,3 The world production of graphite in 2012 was over a million metric tons. ${ }^{3}$ Metallic properties (high electric and thermal conductivities) and non-metallic properties (high thermal resistance, inertness, and lubricity) of graphite ${ }^{3,10}$ make it as an essential material for refractories, ${ }^{14}$ automobiles, ${ }^{15}$ lithiumion batteries, ${ }^{17}$ fuel cells, ${ }^{18}$ solar cells,${ }^{19}$ nuclear reactors, ${ }^{20}$ and graphene production. ${ }^{21}$ Owing to the limited reserve of natural graphite and the ever-increasing demand in energy applications, graphite has been designated as a "supply risk material" by the British Geological Survey ${ }^{22}$ (graphite is ranked 9th in terms of supply risk, higher than the platinum group elements).

Synthetic graphite can fill the gap between supply and demand, but there has been not much research on the process of making synthetic graphite. The common manufacturing process used to synthesize graphite, known as Acheson process, ${ }^{23}$ relies on thermal heating of amorphous carbon to $3000{ }^{\circ} \mathrm{C}$. Although the energy requirements are enormous, (typical processing time is 2 weeks) the Acheson process remains the major industrial process since its invention in the $1960 \mathrm{~s} .{ }^{3}$ Several researches about different way of graphitization like current-induced annealing ${ }^{24}$ or low-temperature graphitization ${ }^{25}$ were tried, but full-graphitization of bulk carbon in energy and time efficient

${ }^{a}$ Department of Chemical Engineering, Pohang University of Science \& Technology, 77 Cheongam-Ro, Nam-Gu, Pohang, Gyeongbuk, 790-784, South Korea. E-mail: ce20047@postech.ac.kr

${ }^{b}$ Carbon Convergence Materials Research Center, Korea Institute of Science and Technology, Eunha-ri San 101, Bongdong-eup, Wanju-gun, Jeollabuk-do 565-905, South Korea

$\dagger$ Electronic supplementary information (ESI) available. See DOI: 10.1039/c6ra01989g method has not been reported. Therefore, a novel and energyefficient graphitization process is highly desirable.

Electromagnetic waves deliver energy as radiation without being dissipated through heat transfer medium. ${ }^{26}$ A representative example is the kitchen microwave oven, which can heat food products much faster than thermal heating. Microwave heating has been considered as a candidate for an energy-efficient graphitization method. Several reports have described the heating of carbon materials using microwaves, for example, to regenerate activated carbon,,$^{27-32}$ weld carbon nanotube/polymer composites, ${ }^{33-37}$ and exfoliate graphite. ${ }^{38-45}$ Full graphitization by microwave heating, however, has yet to be achieved. ${ }^{7}$

Besides, the mechanism of microwave heating of solid carbons has not been fully understood. The mechanism underlying microwave heating can vary, depending upon the properties of the energy-absorbing material. Although dielectric relaxation is responsible for heating polar molecules, such as water, a very different heating mechanism functions in electron-rich solids, such as metals (free electrons) or solid carbon materials ( $\pi$-electrons). It has long been proposed that interfacial polarization, that is, Maxwell-Wagner-Sillars (MWS) polarization, is the main mechanism in operation during the microwave heating of solid carbon materials; however, no in-depth studies have tested the validity of this supposition. ${ }^{\mathbf{4 6 , 4 7}}$

Here, we report a novel method of graphitization using microwave heating with catalyst. Although microwave heating has been reported previously, full graphitization of amorphous carbon powders is achieved for the first time by adding catalyst precursors to the amorphous carbon powders. In addition, the mechanism of microwave heating of solid carbons was investigated. We show that MWS polarization is inappropriate for explaining the heating phenomena observed in solid carbon upon microwave irradiation. 


\section{Experimental}

Materials

Activated carbon powders (product name: G60, KB) and nickel(II) chloride (98\%) were obtained from Sigma-Aldrich, USA. Graphite powder was obtained from Samchun Chemical, Korea. The characteristics of activated carbons used in this work are listed in Table $\mathrm{S} 1$ in ESI. $\dagger$ Elemental analysis was performed using varioMICRO CHNS, and other characteristics were obtained from the technical information supplied by Sigma-Aldrich.

\section{Microwave graphitization}

Microwave graphitization was achieved using the experimental system described in our previous works. ${ }^{48}$ The system consisted of a magnetron, an isolator, a directional coupler, an auto tuner, a quartz reactor, and gas feeders. Impregnated carbon powders were loaded onto the quartz boat, then placed in the quartz reactor. The temperature was measured using an infrared (IR) thermometer, MR1SC, manufactured by Raytek.

Nickel chloride $\left(\mathrm{NiCl}_{2}\right)$ was impregnated with $\mathrm{G} 60$ and $\mathrm{KB}$ in ethanol. During the impregnation step, $6 \mathrm{mmol} \mathrm{NiCl}_{2}$ (per $\mathrm{g}$ carbon powder) were stirred for 5 or more hours at room temperature until all solvents were evaporated. The sample was subsequently dried at $75{ }^{\circ} \mathrm{C}$ for 24 hours.

Next, $1000 \mathrm{~W}$ and $1400 \mathrm{~W}$ microwave radiation was directed onto the G60 and KB samples, respectively. The reactor was filled with argon gas under normal pressure prior to turning on the microwave generator. The flow rate of the argon gas during microwave irradiation was $100 \mathrm{sccm}$.

The carbon powder underwent structural changes before and after microwave irradiation, as observed using X-ray diffraction (XRD) methods with a $\mathrm{CuK}_{\alpha}$ radiation source, Raman spectroscopy (Horiba Jobin Yvon, LabRam HR) using the $514.5 \mathrm{~nm}$ line of an argon ion laser, and by transmission electron microscopy (HRTEM, JEOL JEM-2200FS with image Cs-corrector).

\section{Thermal graphitization}

Thermal graphitization was achieved using lab-scale thermal furnace, Lindberg blue M. The experiment was proceeded at 1000 ${ }^{\circ} \mathrm{C}$ with $\mathrm{NiCl}_{2}$-impregnated $\mathrm{KB}$ for varying time. Since the heat up time for reaching $1000^{\circ} \mathrm{C}$ is $30 \mathrm{~min}$, total processing time of 5,10 , and $30 \mathrm{~min}$ heat treated sample was 35,40 , and $60 \mathrm{~min}$ respectively. The carbon powder underwent structural changes before and after microwave irradiation, as observed using X-ray diffraction (XRD) methods with a $\mathrm{CuK}_{\alpha}$ radiation source.

\section{Dielectric loss factor calculation}

The validity of MWS polarization (also known as interfacial polarization) as a heating mechanism of solid carbons under microwave irradiation was investigated by comparing dielectric loss factor $\left(\varepsilon^{\prime \prime}\right)$.

The average power absorbed by the material $\left(P_{\mathrm{av}}\right)$ is directly proportional to the effective dielectric loss factor $\left(\varepsilon_{\text {eff }}\right)$, as shown in eqn (1), and the effective dielectric loss factor can be separated into the various polarization and conduction components using eqn (2).

$$
\begin{gathered}
P_{\mathrm{av}}=2 \pi f \varepsilon_{0} \varepsilon^{\prime \prime} \text { eff }_{\text {rms }}+2 \pi f \mu_{0} \mu^{\prime \prime}{ }_{\text {eff }} H^{2}{ }_{\text {rms }} \\
\varepsilon_{\text {eff }}^{\prime \prime}=\varepsilon^{\prime \prime}{ }_{\text {dipolar }}+\varepsilon^{\prime \prime}{ }_{\text {electronic }}+\varepsilon^{\prime \prime}{ }_{\text {atomic }}+\varepsilon^{\prime \prime}{ }_{\text {MWs }}+\varepsilon^{\prime \prime} \text { conduction }
\end{gathered}
$$

Therefore, the calculated dielectric loss factor based on the MWS polarization theory ( $\left.\varepsilon^{\prime \prime}{ }_{\text {Mws }}\right)$ should have similar value with those of good microwave absorbers if the theory has meaningful impact on the heating of solid carbon under microwave irradiation.

$\varepsilon^{\prime \prime}{ }_{\text {Mws }}$ of solid carbon was calculated using eqn (3) from the original Sillars' paper. ${ }^{13}$

$$
\varepsilon^{\prime \prime}=\frac{2 \varepsilon^{\prime}{ }_{1} N \pi f \tau}{1+(2 \pi f)^{2} \tau^{2}}
$$

here, $\tau$ and $N$ are described by eqn (4) and (5),

$$
\begin{gathered}
\tau=\frac{\varepsilon_{1}^{\prime}(n-1)+\varepsilon_{2}^{\prime}}{4 \pi \sigma_{2}} \\
N=q \frac{n^{2} \varepsilon_{1}^{\prime}}{\varepsilon_{1}^{\prime}{ }_{1}(n-1)+\varepsilon^{\prime}}
\end{gathered}
$$

where $\varepsilon^{\prime}{ }_{1}$ refers to the dielectric constant of the insulating medium, $\varepsilon_{2}^{\prime}$ refers to the dielectric constant of the conducting medium, $\sigma_{2}$ refers to the conductivity of the conducting medium, $q$ refers to the volume fraction of the conducting medium, $n$ refers to the eccentricity of the spheroid, and $f$ refers to the frequency of the electric field.

In the calculation, a composite material that consists of carbons with low electrical conductivity (e.g. diamond and polymer) and carbons with high electrical conductivity (e.g. graphite and graphene) was assumed. Proper value or range of the variables for the material are summarized in Table 1 . The calculation was proceeded for much wider range.

\section{Results and discussion}

\section{Microwave graphitization of activated carbon powders}

Full graphitization using microwave heating has not been achieved since the temperature increase reaches saturation below $2000{ }^{\circ} \mathrm{C},{ }^{7,49}$ far below the graphitization temperature of

Table 1 Proper value/range of variables and the range of calculation

\begin{tabular}{lll}
\hline Variable & Proper value/range & The range of calculation \\
\hline$\varepsilon^{\prime}{ }_{1}$ & $1-10$ (ref. 5 and 6) & $0-30$ \\
$\varepsilon^{\prime}{ }_{2}$ & $12-15$ (ref. 5) & $0-30$ \\
$\sigma_{2}$ & $10^{3}$ to $10^{6} \mathrm{~S} \mathrm{~cm}^{-1}$ & $10^{3}$ to $10^{6} \mathrm{~S} \mathrm{~cm}^{-1}$ \\
& (ref. 8 and 9) & \\
$q$ & $0-1$ & $0-1$ \\
$n$ & $3-10^{a}$ & $3-30$ \\
$f$ & $915 \mathrm{MHz}, 2.45 \mathrm{GHz}$ & $10^{5}$ to $10^{20} \mathrm{~Hz}$
\end{tabular}

${ }^{a}$ Crystallite height $\left(L_{\mathrm{c}}\right)$ and the crystallite diameter $\left(L_{\mathrm{a}}\right)$ generally have similar values ${ }^{11}$ (meaning that $n$ may be varied over a moderate range from 3 to 10 (ref. 13)). 


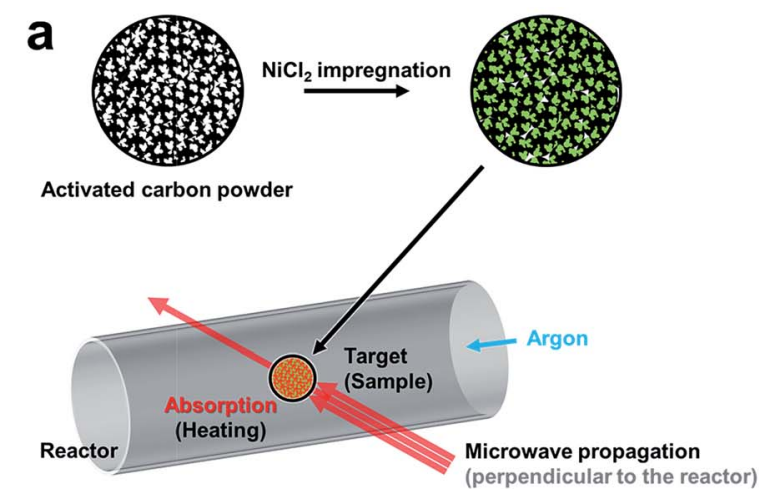

\section{b 1. Microwave irradiation}
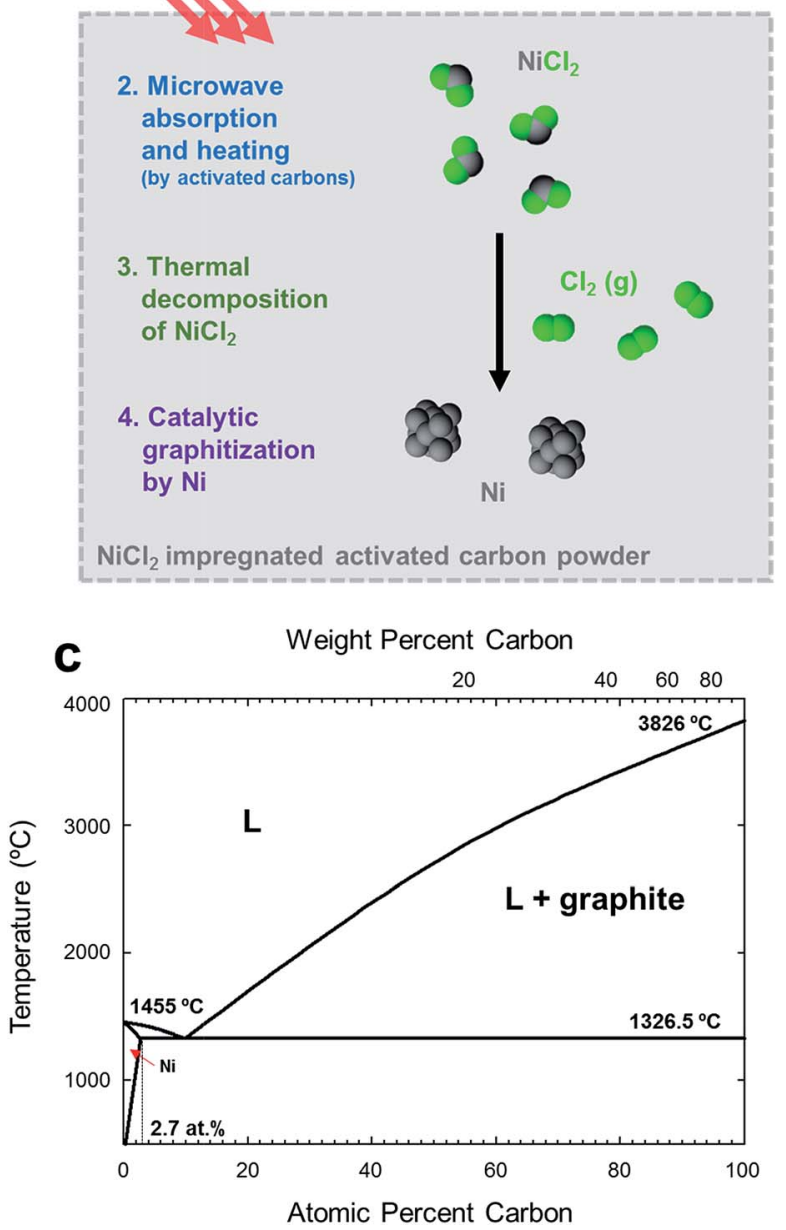

Fig. 1 Illustration of microwave graphitization. (a) Experimental procedure. Microwave was irradiated to the activated carbon powders after impregnating nickel chloride. (b) Detail description of microwave graphitization with metal catalyst. Nickel chloride was first decomposed to form nickel catalyst particles, and then amorphous carbon was transformed to graphite with the help of metal catalyst. (c) Nickelcarbon phase diagram. ${ }^{4}$ Carbide phase in the system is not indicated.

$3000{ }^{\circ} \mathrm{C}$. In our experiments, amorphous carbon powders were first impregnated with metal catalysts, then they were graphitized using microwave heating (Fig. 1a), being inspired by the fact that precipitation of the graphitic carbon on the metal catalysts occurred at around $1000{ }^{\circ} \mathrm{C}$ during thermal heating. ${ }^{50-52}$

The process of catalytic graphitization is shown in Fig. 1b. First, the energy of irradiated microwave is absorbed by amorphous carbon and the temperature of sample reached over $1000{ }^{\circ} \mathrm{C}$ (Fig. S1 and Table S2, ESI $\dagger$ ). At such high temperature, $\mathrm{NiCl}_{2}$ and $\mathrm{NiCl}_{2} \cdot 6 \mathrm{H}_{2} \mathrm{O}$ (Fig. S2, ESI $\dagger$ ) undergoes dehydration and decomposition, ${ }^{53}$ generating nickel particles (Fig. S3 and $\mathrm{S} 4, \mathrm{ESI} \dagger$ ). The phase diagram of $\mathrm{Ni}$ and $\mathrm{C}$ (Fig. 4c) displays that a certain amount of carbon can be dissolved in nickel at high temperature (maximum solubility of carbon in $\mathrm{Ni}$ is about 2.7 at $\%$ at $1326.5{ }^{\circ} \mathrm{C}$ ). When the $\mathrm{Ni}-\mathrm{C}$ solution is supersaturated, the dissolved carbon can be precipitated as a form of graphite spontaneously because the Gibbs free energy change of disordered carbon to graphite is negative. ${ }^{54}$ The Ni-C solution can reach supersaturation either by (i) additional carbon diffusion into the catalyst or (ii) decrease of solubility by lowered temperature. ${ }^{55}$ The catalyst particles migrate throughout the carbon matrix and transform relatively disordered carbon into crystalline graphite until they are deactivated. Besides the catalytic graphitization mechanism, it was also suggested that the meta-stable carbide, $\mathrm{Ni}_{3} \mathrm{C}$ contributes to the precipitation of graphite. ${ }^{4,51,56}$
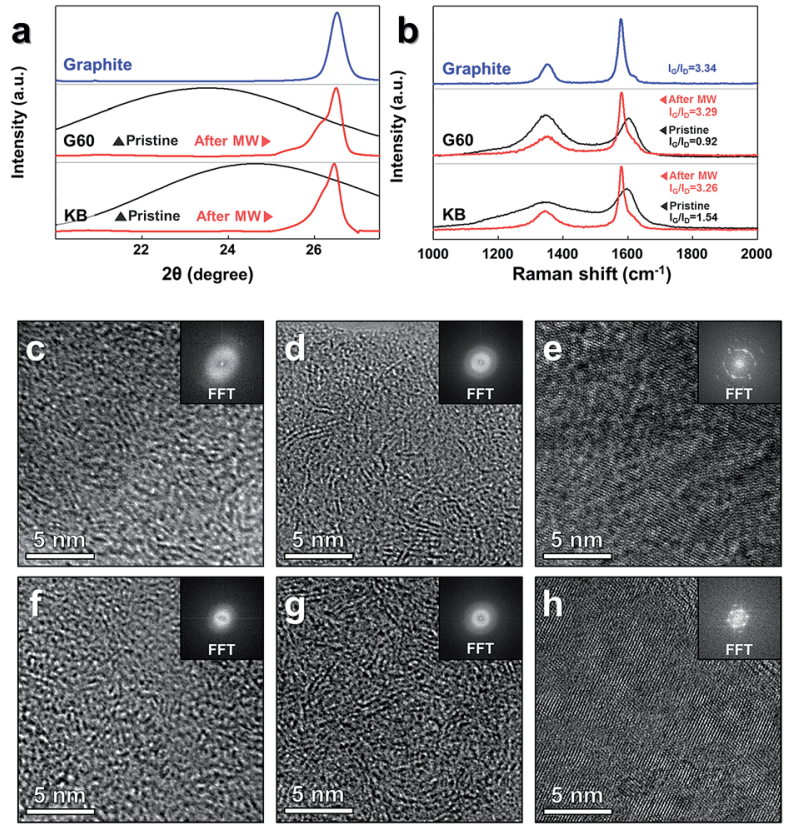

Fig. 2 Evolution of the structural ordering during microwave heating. (a) XRD patterns near the (002) peak of graphite, G60 and KB before and after microwave heating. The position and shape of the (002) peaks of G60 and KB nearly coincided with the peak of the reference graphite powder after microwave heating. (b) Raman spectra of G60 and $\mathrm{KB}$ before and after microwave heating. The $I_{\mathrm{G}} / I_{\mathrm{D}}$ ratios of $\mathrm{G} 60$ and KB increased to 3.29 and 3.26 respectively, values that were nearly indistinguishable from those obtained from the reference graphite powder. (c-h) TEM image and FFT image (inset) of pristine G60 (c), after microwave heating without catalyst (d) and after microwave heating with catalyst (e). TEM image and FFT image (inset) of pristine KB (f), after microwave heating without catalyst (g) and after microwave heating with catalyst (h). 
The changes in the crystalline structure were confirmed by measuring the XRD patterns (Fig. 2a). The position of the (002) peak in the XRD pattern was directly related to the $d$-spacing $\left(d_{002}\right)$ of the carbon sample. ${ }^{10,57}$ The changes in the (002) peak were quantified by analyzing a magnified view of the XRD data collected around the (002) peak (full XRD patterns in Fig. S3, ESI $\dagger$ ). The value of $2 \theta$ obtained at the (002) G60 peak was shifted from $23.45^{\circ}$ to $26.50^{\circ}$, and the value of $\mathrm{KB}$ was shifted from $24.57^{\circ}$ to $26.46^{\circ}$ under microwave heating in the presence of the catalysts. The $d$-spacings of G60 and the value of KB calculated according to Bragg's law were $3.3610 \AA$ And $3.3662 \AA$, respectively, similar to the $d$-spacing of the reference graphite powder (3.3612 $\AA$ ) (detailed information about the $d$-spacing calculations is provided in Table S3, ESI $\dagger$ ). The shapes of the (002) peaks collected from both samples changed dramatically after microwave heating and became nearly identical to the shape obtained from the reference graphite powder. These results indicated that amorphous carbon atoms were effectively graphitized under microwave heating in the presence of the metal catalyst. In the absence of the metal catalyst, however, distinctive changes were not observed in either sample under microwave heating (Fig. S5, ESI†).

The Raman spectra (Fig. 2b) further supported the conclusions obtained from the XRD study. Perfect graphite was characterized by a single high-frequency band at $1582 \mathrm{~cm}^{-1}$, which corresponded to the $\mathrm{E}_{2} \mathrm{~g}_{2}$ mode, or G-band. Disordered carbon was characterized by another broad band at approximately $1350 \mathrm{~cm}^{-1}$, which corresponded to the D-band. ${ }^{58}$ The ratio of the integrated or maximum intensity of the G-band to the D-band is commonly used to characterize the different types of carbon-based materials. ${ }^{\mathbf{5 9 , 6 0}}$ Higher fractions of the graphitic region produced larger $I_{\mathrm{G}} / I_{\mathrm{D}}$ ratios. The $I_{\mathrm{G}} / I_{\mathrm{D}}$ ratios of the pristine samples were 0.92 (G60) and 1.54 (KB), and these values increased to 3.29 (G60) and 3.26 (KB) after microwave heating in the presence of the catalysts. These values were similar to the value obtained from the reference graphite powder (3.34), further supporting the conclusion that the amorphous carbon powders were indeed transformed into graphitic carbon under microwave heating in the presence of the catalysts.

Transmission electron microscopy (TEM) images reconfirmed the transformation from an amorphous structure to a graphitic structure (Fig. 2c-h). Prior to microwave heating, G60 and KB only exhibited disordered microstructures typical of amorphous carbon. The fast Fourier transform (FFT) images showed hollow circles. On the other hand, completely different TEM and FFT images were obtained after microwave heating in the presence of the catalysts. The crystalline ordering was apparent throughout the entire sample, and hexagonal patterns appeared. Multiple sets of dots with six-fold symmetry were observed in the FFT images. These results indicated that multilayer graphene sheets developed during microwave heating in the presence of the catalysts.

\section{Comparison with thermal graphitization}

Interlayer spacing of various carbon materials as a function of the processing temperature is summarized in Fig. 3a. The interlayer spacing, or $d$-spacing, refer to the distance between
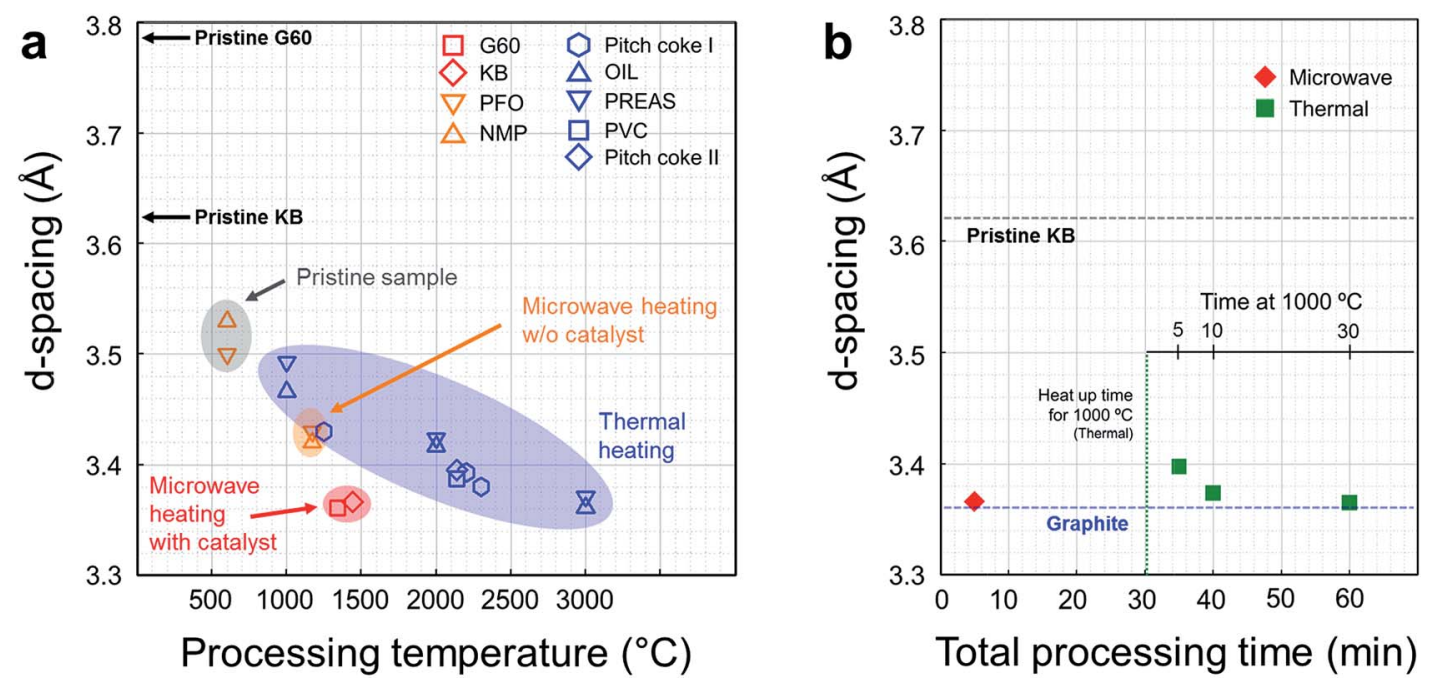

Fig. 3 Comparison between microwave graphitization and thermal graphitization. (a) $d$-Spacing in various carbons as a function of the processing temperature (G60 and KB: activated carbon powders, PFO: pyrolyzed isotropic pitch from fuel oil,7 NMP: naphthalene-derived mesophase pitch. ${ }^{7}$ Pitch coke I: carbonized coal-tar pitch, ${ }^{12}$ OIL: cyclohexane soluble fraction of benzene soluble part of coal liquid pitch, ${ }^{11}$ PREAS: vacuum dried pre-asphaltenes of the benzene-insoluble part of the coal liquid pitch, ${ }^{11}$ PVC: polyvinyl chloride, ${ }^{16}$ pitch coke II: carbonized pitch ${ }^{16}$ ). Conventional graphitization using resistive heating requires high temperatures exceeding 3000 and extremely long processing times (1-25 h, see the ESI, Table S2 $\dagger$ ). In the absence of a catalyst, microwave heating changed the crystallinity of the carbon powders over a short period of time; however, full graphitization was not achieved (orange shade). In this work, effective and efficient graphitization was achieved using microwave heating and catalyst precursor. The $d$-spacing in the activated carbon samples changed to $3.36 \AA$ within 5 minutes of processing time (red symbols). (b) Comparison with thermal catalytic graphitization using lab-scale thermal furnace. Thermal graphitization proceeded for 5 , 10, and $30 \mathrm{~min}$ at $1000^{\circ} \mathrm{C}$, and microwave graphitization proceeded for $5 \mathrm{~min}$ at a $1400 \mathrm{~W}$ microwave power. The $d$-spacing of the raw material (KB) and graphite are presented for reference purposes. 
adjacent graphene layers. This parameter corresponds to the degree of graphitization. ${ }^{\mathbf{1 1}}$ In general, the $d$-spacing of a carbon material tends to decrease as the processing temperature increases during thermal heating (blue symbols). Microwave heating can decrease the $d$-spacing more efficiently than thermal heating at the same processing temperature, although the lowest achievable value is only $3.42 \AA$ without the help of a catalyst (orange shade). This $d$-spacing is equivalent to that obtained under thermal heating at $2000{ }^{\circ} \mathrm{C}$ (Table S4, ESI $\dagger$ ). In the presence of the metal catalyst, however, full graphitization of the carbon powders was achieved with a $d$-spacing of $3.3610 \AA$ (red symbols, this work).

The merits of the microwave graphitization in terms of time required to reach completion were examined by comparing the microwave process conditions to the thermal heating conditions, applied to a $\mathrm{NiCl}_{2}$-impregnated $\mathrm{KB}$ sample for different heat treatment periods (Fig. 3b). Microwave graphitization displayed tremendous advantages in terms of the time required to complete the process. Only $5 \mathrm{~min}$ of microwave irradiation provided similar structural changes as $60 \mathrm{~min}$ of the conventional process (thermal graphitization). Thermal graphitization requires $30 \mathrm{~min}$ for heat up to $1000{ }^{\circ} \mathrm{C}$, so additional $30 \mathrm{~min}$ is required for the graphitization, whereas heat up over $1000{ }^{\circ} \mathrm{C}$ and graphitization was achieved in $230 \mathrm{~s}$ and $70 \mathrm{~s}$ respectively (Fig S1 and Table S5, ESI $\dagger$ ).

We demonstrated the efficiency of microwave heating over conventional heating in graphitization at the lab-scale experiments. The scale-up of our experiment can be done based on the understanding of penetration depth. The definition of penetration depth $\left(d_{\mathrm{p}}\right)$ is the distance from the surface to a certain point where magnitude of field strength decreases to $1 / e$ of original magnitude at the surface. ${ }^{61}$ The penetration depth of electric field is expressed as the following equation.

$$
d_{\mathrm{p}}=1 /\left\{2 \pi f\left(\frac{\mu_{0} \mu^{\prime} \varepsilon_{0} \varepsilon^{\prime}}{2}\right)^{1 / 2}\left[\left(1+(\tan \delta)^{2}\right)^{1 / 2}-1\right]^{1 / 2}\right\}
$$

here, $\varepsilon_{0}$ refers to the absolute permittivity, $\varepsilon^{\prime}$ refers to the relative permittivity, $\mu_{0}$ refers to the absolute permeability, $\mu^{\prime}$ refers to the relative permeability and $\tan \delta$ refers to the loss tangent. ${ }^{62}$

The importance of penetration depth in scale-up of microwave reactor is represented in Fig. 4a. Heat is generated from the whole volume of target material when the microwave penetration depth is bigger than the scale of target material $\left(l_{\text {target }}\right)$. When the scale of target material is much bigger than the microwave penetration depth, however, heat is hardly generated from the inside of the material. Only surface region is heated by microwave radiation and the rest of the material is heated by conduction from the surface.

Crystalline carbon materials have very short penetration depth, typically several microns, ${ }^{47}$ so most benefits of microwave heating vanish with bulky target material. Nevertheless, if we have an accurate understanding of the penetration depth, scale-up of microwave reactor which still has the advantages of microwave heating is possible. A hint for the scale-up of microwave reactor can be obtained from the plate-type reactor which is commercially available (Fig. S7, ESI $\dagger$ ). This reactor was
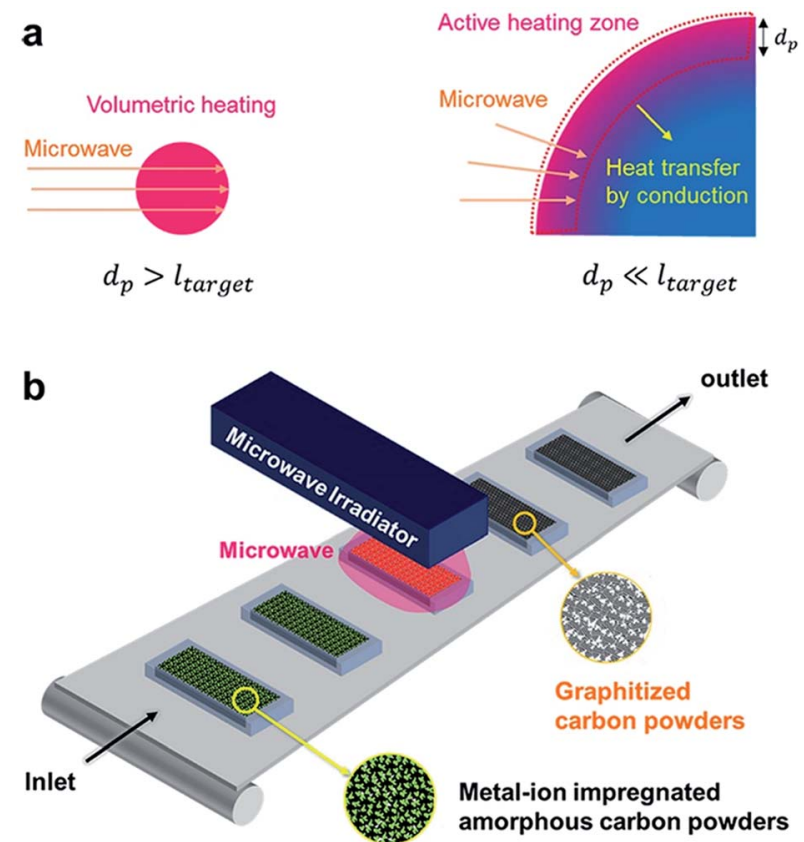

Fig. 4 Scale-up of microwave reactor. (a) The effect of penetration depth on microwave heating. (b) Illustration of conveyor belt type reactor with microwave irradiator.

introduced to maximize the heat delivery from the heating fluid to the reactants. A similar approach can be applied to the scaleup of a microwave reactor.

An easy solution to the problem of short penetration depth is the use of conveyer belt type reactor with small size carbon powders (Fig. 4b). A tumbling-bed reactor or a fluidized-bed reactor can also be used because each carbon powder is separated from others. This kind of reactor design enables high productivity, and prevents the problem of short penetration depth at the same time.

\section{Verification of heating mechanism}

In spite of the effectiveness and efficiency of microwave heating of solid carbons shown in this work, the study about the heating mechanism is still much to be required. Until now, the mechanisms underlying microwave heating of solid carbons are understood as Maxwell-Wagner-Sillars (MWS) polarization $^{\mathbf{4 6 , 6 3 , 6 4}}$ or joule heating reference. However, there has been no in-depth study about the validity of the MWS mechanism. Therefore, we investigated the influence of MWS polarization over microwave heating of solid carbons by dielectric loss factor $\left(\varepsilon^{\prime \prime}\right)$ calculation. As explained in the experimental part, the average power absorbed by the material under electromagnetic wave is directly proportional to the effective dielectric loss factor ( $\varepsilon^{\prime \prime}$ eff $)$ of the material, and $\varepsilon^{\prime \prime}$ eff consists of various polarizations and conduction components. Therefore, if MWS polarization is responsible for the microwave heating of solid carbons, the calculated dielectric loss factor based on MWS polarization theory should have similar or larger value than the dielectric loss factor of good microwave absorbers, because solid carbons 
show extreme heating phenomena under microwave irradiation.

Among the six variables in the eqn (3) which was used in the dielectric loss factor calculation, $n$ and $q$ only change the numerical value, and do not change the tendency. The dielectric loss factor increases as either $n$ or $q$ increases (Fig. S8-S10 $\dagger^{\dagger}$ ). Thus, in the result part, the influence of the other four variables on the dielectric loss factor was examined. Then, the maximum value in the range of the properties of solid carbons was obtained and compared with the values of other materials for judging whether the MWS polarization is proper or not for the microwave heating mechanism of solid carbons.

At first, the dielectric loss factor ( $\varepsilon^{\prime \prime}{ }_{\text {Mws }}$ ) was calculated by varying the dielectric constant of solid carbons $\left(\varepsilon^{\prime}{ }_{1}\right.$ and $\left.\varepsilon^{\prime}{ }_{2}\right)$. The dielectric constant of conducting region $\left(\varepsilon^{\prime}{ }_{1}\right)$ has little effect on $\varepsilon^{\prime \prime}{ }_{\text {MWS }}$, and $\varepsilon^{\prime \prime}{ }_{\text {Mws }}$ increases with increasing dielectric loss factor of insulating region $\left(\varepsilon_{2}^{\prime}\right)$ as shown in Fig. 5a. The maximum value of $\varepsilon^{\prime \prime}{ }_{\text {Mws }}$ was 1.1 when $\varepsilon^{\prime}{ }_{1}=30, \sigma_{2}=1000 \mathrm{~S} \mathrm{~cm}^{-1}, q=1, n$ $=30$ and $f=2.45 \mathrm{GHz}$. The maximum value of calculated dielectric loss factor is 1-2 orders smaller than those of the materials known as good microwave absorbers, even though the range of calculation was much wider. It means that the contribution of MWS polarization is very small for the microwave heating of solid carbons.

Next, the influence of conductivity of conducting region $\left(\sigma_{2}\right)$ and the applied frequency $(f)$ was investigated. A bell-shaped curve was obtained when $\varepsilon^{\prime \prime}$ Mws was plotted as a function of $\log$ frequency $\left(\log f\right.$ ) (Fig. 5b). The peak frequency $\left(f_{\text {peak }}\right)$ increased as the electrical conductivity of conducting region $\left(\sigma_{2}\right)$ increased. The key results are summarized in Table 2. When $\sigma_{2}$
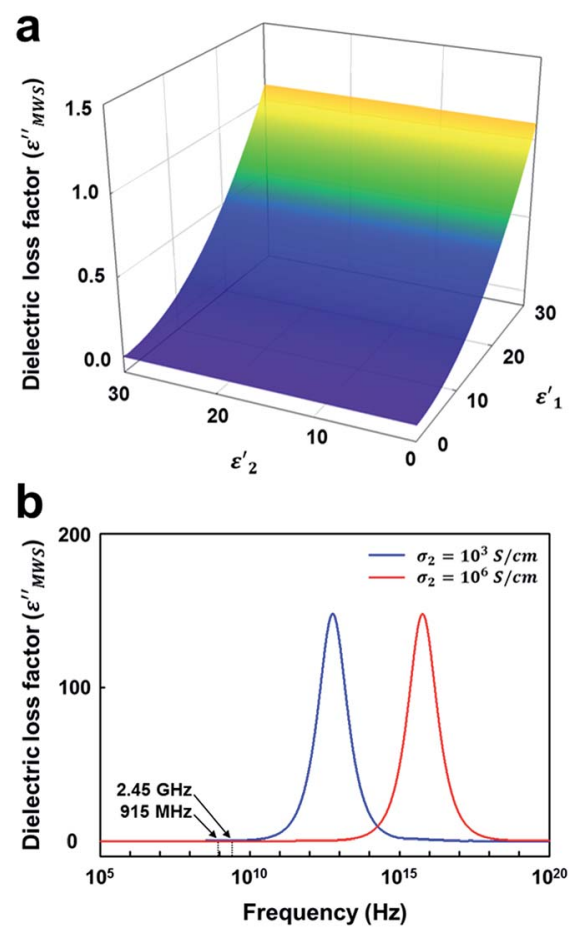

Fig. 5 The influence of dielectric constants $\left(\varepsilon_{1}^{\prime}\right.$ and $\left.\varepsilon_{1}^{\prime}{ }_{1}\right)$ on the dielectric loss factor of solid carbon based on MWS polarization ( $\varepsilon^{\prime \prime}$ MWS $)$. $\left(\sigma_{2}=1000 \mathrm{~S} \mathrm{~cm}^{-1}, q=1\right.$, and $n=30$ and $f=2.45 \mathrm{GHz}$ ).
Table 2 The peak frequency $\left(f_{\text {peak }}\right)$ and the calculated dielectric loss factor $\left(\varepsilon^{\prime \prime}\right.$ frequency) with different conductivity $\left(\sigma_{2}\right)$ and frequency. $\left(\varepsilon_{1}^{\prime}=\right.$ $\varepsilon_{2}^{\prime}=30, q=1$ and $n=30$ )

\begin{tabular}{llll}
\hline$\sigma_{2}\left(\mathrm{~S} \mathrm{~cm}^{-1}\right)$ & $f_{\text {peak }}$ & $\varepsilon^{\prime \prime}{ }_{915 \mathrm{MHz}}$ & $\varepsilon^{\prime \prime}{ }_{2.45 \mathrm{GHz}}$ \\
\hline $10^{3}$ & $2 \times 10^{12} \mathrm{~Hz}(2 \mathrm{THz})$ & 0.4117 & 1.1025 \\
$10^{6}$ & $2 \times 10^{15} \mathrm{~Hz}(2 \mathrm{PHz})$ & 0.0004 & 0.0011
\end{tabular}

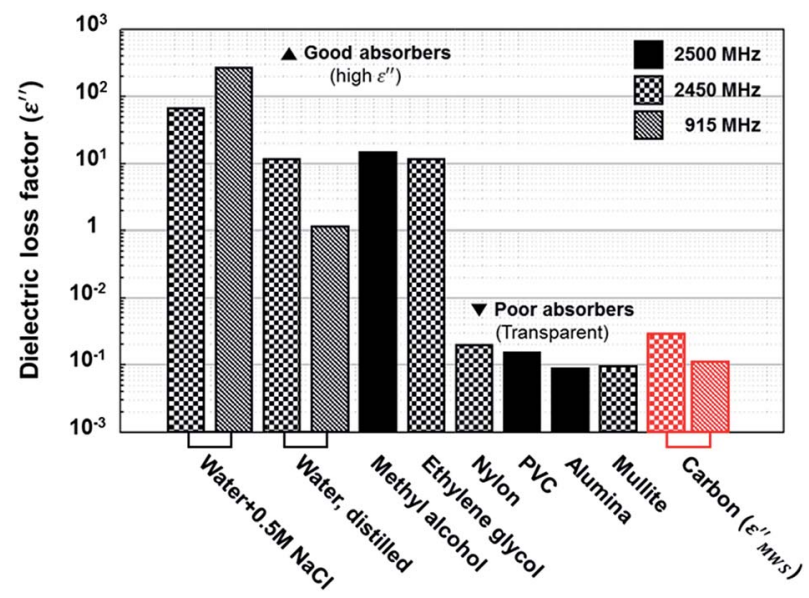

Fig. 6 Dielectric loss factor comparison. ${ }^{1}$ Calculated dielectric loss factors of solid carbon based on MWS polarization ( $\left.\varepsilon^{\prime \prime}{ }_{M W S}\right)$ are located at poor absorber region, where microwave transparent materials are exist.

$=10^{3} \mathrm{~S} \mathrm{~cm}^{-1}$ (around the electrical conductivity of graphite ${ }^{58}$ ), $f_{\text {peak }}$ was $2 \mathrm{THz}$, and the dielectric loss factor at $915 \mathrm{MHz}$ and $2.45 \mathrm{GHz}$ was 0.4117 and 1.1025 respectively. When $\sigma_{2}=10^{6} \mathrm{~S}$ $\mathrm{cm}^{-1}$ (a much larger value than those of conducting solid carbons $\left.^{8,9}\right), f_{\text {peak }}$ was $2 \mathrm{PHz}$, and the dielectric loss factor at $915 \mathrm{MHz}$ and $2.45 \mathrm{GHz}$ was 0.0004 and 0.0011 respectively. In other words, $f_{\text {peak }}$ of solid carbon material is present at a much higher frequency than microwave frequencies. Moreover, $\varepsilon^{\prime \prime}$ Mws is very small at $915 \mathrm{MHz}$ and $2.45 \mathrm{GHz}$, typical frequencies in microwave heating. The results indicate that the contribution of MWS polarization to the heating of solid carbons in the range of microwave frequencies is very small or almost negligible.

Finally, the maximum value of calculated dielectric loss factor based on MWS polarization in the proper range for solid carbons was compared to the value of other materials. As discussed above, $\varepsilon^{\prime \prime}{ }_{\text {Mws }}$ has larger value when $\varepsilon^{\prime}{ }_{1}, q$, and $n$ become larger, and $\sigma_{2}$ becomes smaller. The maximum values within the proper range shown in Table 1 are plotted in Fig. 6 with the dielectric loss factor of other materials. The maximum value of calculated dielectric loss factor for solid carbons based on MWS polarization was 0.0114 under $915 \mathrm{MHz}$ and 0.0306 under 2.45 GHz. These values are far below than the values of the materials known as good microwave absorbers (water $+0.5 \mathrm{M}$ NaCl: 269, methyl alcohol: 15). Actually, they are similar to the values of poor microwave absorbers (alumina: 0.009, mullite: 0.0098). Therefore, it seems inadequate to explain the extreme 
heating phenomena of solid carbons under microwave irradiation relying only on MWS polarization.

\section{Conclusions}

In conclusion, we report the full graphitization of amorphous carbon powders by modified microwave heating with the help of a metal catalyst. Because microwave graphitization is much more efficient, in terms of energy and time requirements, than the industrial graphitization method currently in use, this method is expected to provide an alternative approach to producing graphite, which is considered to have a high supply risk. Because the economic gains of our method are considerable, this approach has the potential to revolutionize the graphite industry. We also examined the validity of the Maxwell-Wagner-Sillars polarization, which has long been accepted as a mechanism underlying microwave heating in solid carbons. The dielectric loss factor calculations over a wide range of parameter space revealed that the MWS polarization is certainly inadequate for describing the microwave heating mechanism in solid carbons.

\section{Acknowledgements}

The present work was supported by Korea Science and Engineering Foundation (KOSEF) grant funded by the Korean government (MEST) (Grant No. 2015-021048). We also acknowledge the Research Institute of Industrial Science \& Technology for financial support. This work was also supported by NRF (National Research Foundation of Korea) Grant funded by the Korean Government (NRF-2011-Global Ph.D. Fellowship Program).

\section{Notes and references}

1 M. Gupta and E. W. W. Leong, Microwaves and Metals, Jonh Wiley \& Sons(Asia) Pte Ltd, Singapore, 2007.

2 A. Zurutuza and C. Marinelli, Nat. Nanotechnol., 2014, 9, 730734.

3 Graphite, http://minerals.usgs.gov/minerals/pubs/commodity/ graphite/myb1-2013-graph.pdf, (accessed 23.10.2015).

4 M. Singleton and P. Nash, J. Phase Equilib., 1989, 10, 121126.

5 Dielectric Constant Values, http://www.clippercontrols.com/ pages/Dielectric-Constant-Values.html\#D, (accessed 22.05.2015, 2015).

6 Z. Ahmad, Polymer Dielectric Materials, InTech, 2012.

7 K. S. Yang, Y. J. Yoon, M. S. Lee, W. J. Lee and J. H. Kim, Carbon, 2002, 40, 897-903.

8 H. O. Pierson, Handbook of carbon, graphite, diamond, and fullerenes: properties, processing, and applications, Noyes Publications, Park Ridge, N.J., U.S.A., 1993.

9 J.-H. Chen, C. Jang, S. Xiao, M. Ishigami and M. S. Fuhrer, Nat. Nanotechnol., 2008, 3, 206-209.

$10 \mathrm{~K}$. Kinoshita, Carbon: electrochemical and physicochemical properties, John Wiley \& Sons, Inc., Canada, 1988.

11 U. Świetlik, S. Jasieńko and A. Wolski, Carbon, 1993, 31, 461466.
12 K. Kawamura and R. Bragg, Carbon, 1986, 24, 301-309.

13 R. W. Sillars, J. Inst. Electr. Eng., 1937, 80, 378-394.

14 W. E. Lee and R. E. Moore, J. Am. Ceram. Soc., 1998, 81, 13851410.

15 B. Gerard, Surf. Coat. Technol., 2006, 201, 2028-2031.

16 R. E. Franklin, Acta Crystallogr., 1951, 4, 253-261.

17 M. Armand and J. M. Tarascon, Nature, 2008, 451, 652-657.

18 B. C. H. Steele and A. Heinzel, Nature, 2001, 414, 345-352.

19 K. Imoto, K. Takahashi, T. Yamaguchi, T. Komura, J.-i. Nakamura and K. Murata, Sol. Energy Mater. Sol. Cells, 2003, 79, 459-469.

20 D. Butler, Nature, 2004, 429, 238-240.

21 Y. Hernandez, V. Nicolosi, M. Lotya, F. M. Blighe, Z. Y. Sun, S. De, I. T. McGovern, B. Holland, M. Byrne, Y. K. Gun'ko, J. J. Boland, P. Niraj, G. Duesberg, S. Krishnamurthy, R. Goodhue, J. Hutchison, V. Scardaci, A. C. Ferrari and J. N. Coleman, Nat. Nanotechnol., 2008, 3, 563-568.

22 Risk List 2012, https:/www.bgs.ac.uk/mineralsuk/statistics/ riskList.html, (accessed 15.05.2015).

23 Kirk-Othmer concise encyclopedia of chemical technology, Wiley-Interscience, Hoboken, N.J., 5th edn., 2007.

24 A. Barreiro, F. Borrnert, S. M. Avdoshenko, B. Rellinghaus, G. Cuniberti, M. H. Rummeli and L. M. K. Vandersypen, Sci. Rep., 2013, 3, 1115.

25 K. Barbera, L. Frusteri, G. Italiano, L. Spadaro, F. Frusteri, S. Perathoner and G. Centi, Chin. J. Catal., 2014, 35, 869-876.

26 A. C. Metaxas and R. J. Meredith, Industrial microwave heating, $P$. peregrinus on behalf of the Institution of Electrical Engineers, London, UK, 1983.

27 C. Y. Cha and Y. G. Kong, Carbon, 1995, 33, 1141-1146.

28 Y. Kong and C. Y. Cha, Carbon, 1996, 34, 1035-1040.

29 J. A. Menendez, E. M. Menendez, A. Garcia, J. B. Parra and J. J. Pis, J. Microwave Power, 1999, 34, 137-143.

30 J. A. Menendez, E. M. Menendez, M. J. Iglesias, A. Garcia and J. J. Pis, Carbon, 1999, 37, 1115-1121.

31 X. T. Liu, X. Quan, L. L. Bo, S. Chen and Y. Z. Zhao, Carbon, 2004, 42, 415-422.

32 C. O. Ania, J. A. Menendez, J. B. Parra and J. J. Pis, Carbon, 2004, 42, 1383-1387.

33 M. Zhang, S. L. Fang, A. A. Zakhidov, S. B. Lee, A. E. Aliev, C. D. Williams, K. R. Atkinson and R. H. Baughman, Science, 2005, 309, 1215-1219.

34 C. Y. Wang, T. G. Chen, S. C. Chang, S. Y. Cheng and T. S. Chin, Adv. Funct. Mater., 2007, 17, 1979-1983.

35 H. C. Shim, Y. K. Kwak, C. S. Han and S. Kim, Scr. Mater., 2009, 61, 32-35.

36 H. Wang, J. Y. Feng, X. J. Hu and K. M. Ng, Nanotechnology, 2009, 20, 095601.

37 R. Xie, J. Wang, Y. Yang, K. Jiang, Q. Li and S. Fan, Compos. Sci. Technol., 2011, 72, 85-90.

38 V. Sridhar, J. H. Jeon and I. K. Oh, Carbon, 2010, 48, 29532957.

39 O. Y. Kwon, S. W. Choi, K. W. Park and Y. B. Kwon, J. Ind. Eng. Chem., 2003, 9, 743-747.

40 L. M. Viculis, J. J. Mack, O. M. Mayer, H. T. Hahn and R. B. Kaner, J. Mater. Chem., 2005, 15, 974-978. 
41 B. Tryba, A. W. Morawski and M. Inagaki, Carbon, 2005, 43, 2417-2419.

42 E. H. L. Falcao, R. G. Blair, J. J. Mack, L. M. Viculis, C. W. Kwon, M. Bendikov, R. B. Kaner, B. S. Dunn and F. Wudl, Carbon, 2007, 45, 1367-1369.

43 G. Q. Xin, W. Hwang, N. Kim, S. M. Cho and H. Chae, Nanotechnology, 2010, 21, 405201.

44 Y. Geng, Q. B. Zheng and J. K. Kim, J. Nanosci. Nanotechnol., 2011, 11, 1084-1091.

45 C. Xiu-Yun, Int. Nano Lett., 2013, 3, 1-5.

46 J. A. Menendez, A. Arenillas, B. Fidalgo, Y. Fernandez, L. Zubizarreta, E. G. Calvo and J. M. Bermudez, Fuel Process. Technol., 2010, 91, 1-8.

47 T. Kim, J. Lee and K. H. Lee, Carbon letters, 2014, 15, 15-24. 48 D. M. Yoon, B. J. Yoon, K. H. Lee, H. S. Kim and C. G. Park, Carbon, 2006, 44, 1339-1343.

49 P. J. M. Carrott, J. M. V. Nabais, M. M. L. R. Carrott and J. A. Menendez, Microporous Mesoporous Mater., 2001, 47, 243-252.

50 A. Oya and S. Otani, Carbon, 1979, 17, 131-137.

51 A. Oya, J. Mater. Sci., 1982, 17, 309-322.

52 M. Sevilla and A. B. Fuertes, Carbon, 2006, 44, 468-474.
53 S. K. Mishra and S. B. Kanungo, J. Therm. Anal., 1992, 38, 2417-2436.

54 E. Fitzer and B. Kegel, Carbon, 1968, 6, 433-446.

55 J. Lander, H. Kern and A. Beach, J. Appl. Phys., 1952, 23, 1305-1309.

56 C. Mattevi, H. Kim and M. Chhowalla, J. Mater. Chem., 2011, 21, 3324-3334.

57 Y. Hishiyama, M. Inagaki and S. Kimura, Carbon, 1974, 12, 249-258.

58 F. Tuinstra and J. L. Koenig, J. Chem. Phys., 1970, 53, 11261130.

59 D. S. Knight and W. B. White, J. Mater. Res., 1988, 4, 385-393.

60 M. S. Dresselhaus and G. Dresselhaus, Adv. Phys., 1981, 30, 139-326.

61 A. C. Metaxas, Foundations of Electroheat: A Unified Approach, John Wiley \& Sons, Chichester, 1996.

62 M. Gupta and W. L. Wong, Microwaves and metals, John Wiley \& Sons, Singapore, Hoboken, NJ, 2007.

63 E. A. Dawson, G. M. B. Parkes, P. A. Barnes, G. Bond and R. Mao, Carbon, 2008, 46, 220-228.

64 J. M. V. Nabais, P. J. M. Carrott, M. M. L. R. Carrott, A. M. Padre-Eterno, J. A. Menendez, A. Dominguez and A. L. Ortiz, Carbon, 2006, 44, 1158-1165. 